\title{
Elevated serum IL-6 and adiponectin levels are associated with frailty and physical function in Chinese older adults
}

This article was published in the following Dove Press journal:

Clinical Interventions in Aging

\section{Lina Ma}

Guiming Sha

Yaxin Zhang

Yun Li

Department of Geriatrics, Xuanwu Hospital, Capital Medical University, National Clinical Research Center for Geriatric Disorders, Beijing I00053, China
Correspondence: Lina Ma

Department of Geriatrics, Xuanwu Hospital, Capital Medical University, 45 Changchun Street, Xicheng District, Beijing 100053, China

Emailmalina0883@I26.com
Purpose: Frailty is associated with adverse health outcomes. Its biological markers are essential to enhance diagnostic ease and would contribute to surveillance of the condition. Considering the involvement of pro-inflammatory and nutritional states in frailty, we aimed to investigate whether inflammatory mediators and adipokines are associated with frailty and their relationship with physical function.

Patients and methods: We recruited 130 older adults (90 nonfrail participants and 40 frail participants, mean age: $72.80 \pm 8.61$ years) who underwent a comprehensive medical history and frailty assessment. The biochemical indicators (eg, blood urea nitrogen [BUN], high-density lipoprotein [HDL], and hemoglobin [HGB]), insulin pathway (glucose, insulin, and insulin-like growth factor 1 [IGF-1]), circulating inflammatory biomarkers (IL-6, tumor necrosis factor receptor 1, and C-reactive protein), and adipokines (adiponectin, vaspin, and leptin) were compared between the two groups. We further analyzed their correlation with physical function.

Results: Frail older adults showed higher levels of BUN, IL-6, adiponectin, vaspin, and glucose and lower levels of IGF-1, HDL, and HGB compared with nonfrail participants. Serum IL-6 levels were negatively correlated with both grip strength $(P=0.03)$ and gait speed $(P=0.04)$. Levels of circulating adiponectin and leptin were adversely correlated with grip strength $(P=0.01)$ and gait speed $(P=0.03)$, respectively. After adjustment for age and sex, the only markers correlated with physical function were IL-6 $(r=-0.180, P=0.044)$ and adiponectin $(r=-0.195, P=0.029)$.

Conclusion: High levels of IL-6, adiponectin, vaspin, and glucose as well as low levels of IGF-1 were found in frail older adults. Furthermore, IL-6, adiponectin, and leptin levels were negatively correlated with physical function, suggesting that inflammatory mediators and adipokines are biomarkers for frailty and decreased function in older adults.

Keywords: adipokine, adiponectin, biomarker, frailty, inflammation, IL-6

\section{Introduction}

Aging is a leading risk factor for decreased physical function. ${ }^{1}$ As a geriatric syndrome characterized by decreased physical function and functional capacity, frailty is associated with increased vulnerability to stressors, which contribute to a high risk of adverse consequences, such as falls, disability, and mortality in older adults, ${ }^{2}$ as well as the financial burden of long-term care. Owing to its easy application in clinical practice and prognostic value in therapeutic decisions in medical specialists, early identification of a frail status is essential in older adults. Biomarkers may help identify patients who are at a risk of becoming or being frail. However, studies on the biological correlates for frailty in Chinese older adults were limited. 
Low-grade chronic inflammation, a hallmark of aging and mechanism underlying age-related diseases, is associated with the onset or progression of age-related diseases, including frailty ${ }^{3}$ and may serve as a mediator between normal aging and age-related pathological processes. Inflammation has been recognized as a pivotal molecular basis for the pathogenesis of many age-related chronic diseases; increased proinflammatory cytokine levels in the circulation contribute to an inflammatory environment for tissues and organs, ${ }^{4}$ resulting in several frailty-associated pathologies. An interplay of multiple factors including pro-inflammatory state, oxidative stress, and nutritional status was involved in frailty. The adipose tissue has a key role in the integration of systemic metabolism. Adipokines secreted by adipose tissues such as adiponectin were found to be associated with frailty. ${ }^{5}$ Other adipokines such as vaspin and leptin were found to be associated with chronic diseases, but their association with frailty was not yet studied. Finding the biological correlates would allow physicians to identify frailty, monitor the progression and efficacy of interventions, and delay the onset of frailty and occurrence of disability. Thus, we aimed to investigate the association of inflammatory cytokines and adipokines with frailty and their relationship with physical performance in older individuals.

\section{Patients and methods}

\section{Study design and participants}

Data were from cardiovascular health, cognition and aging study (CHCAS). A total of 130 inpatients aged 60-92 (mean age: $72.80 \pm 8.61$ ) years were included in this study: 90 older nonfrail participants and 40 frail older subjects. All patients were from Department of Geriatrics, Xuanwu Hospital, Capital Medical University. Exclusion criteria included infection, acute conditions, severe diseases such as organ dysfunction, corticosteroids, methotrexate, and nonsteroidal anti-inflammatory drugs or other immune-modulating agents. The study was approved by the Institutional Review Board of Xuanwu Hospital, Capital Medical University, and written informed consent was obtained from all participants prior to participation. This study was conducted in accordance with the Declaration of Helsinki.

\section{Data collection}

All participants underwent a comprehensive medical history and frailty measurement by trained staff. Socioeconomic and demographic data on age, sex, marital status, and educational level were collected. Chronic diseases such as hypertension, ischemic heart disease (IHD), diabetes, and stroke were self-reported by the participants. Body mass index (BMI) was calculated as weight $(\mathrm{kg}) /$ height $\left(\mathrm{m}^{2}\right)$. Blood pressure was measured in sitting position with a 10-minute rest. Ejection fraction (EF) was determined using an echocardiogram. The energy level was self-reported, with the total score of 10: 0 as the lowest energy level and 10 as the highest energy level.

\section{Frailty assessment}

Frailty assessment including slowness, fatigue, weight loss, weakness, and low activity was measured according to the Fried frailty phenotype. ${ }^{2}$ Grip strength was assessed according to standardized procedures, and the maximum value of the three measurements was used for analysis. ${ }^{2}$ Walking speed $(\mathrm{m} / \mathrm{s})$ was measured by taking a $20-\mathrm{m}$ walk at a usual pace. The cutoff value of weakness for grip strength and slowness for walking speed was determined as previously reported in Chinese older population. ${ }^{6}$ The total score of frailty is $0-5$. Those with $\geq 3$ were classified as frail, and the others were nonfrail ( 0 , robust; $1-2$, prefrail). Peripheral blood samples were collected after overnight fasting, and serum samples were isolated and stored at $-80^{\circ} \mathrm{C}$ before use.

\section{Biochemical measurement}

Triglyceride (TG), total cholesterol (TC), high-density lipoprotein (HDL), low-density lipoprotein (LDL), uric acid (UA), creatinine ( $\mathrm{Cr}$ ), blood urea nitrogen (BUN), alanine aminotransferase (ALT), aspartate aminotransferase (AST), homocysteine (Hcy), prealbumin (PA), glucose, and insulin were determined using a Hitachi 7600 automatic biochemical analyzer (Hitachi, Tokyo, Japan). White blood cell (WBC) count, red blood cell (RBC) count, platelet (PLT), and hemoglobin (HGB) were determined by standard laboratory methods. Brain-derived neurotrophic factor (BDNF) was measured using ELISA (R\&D Systems, Minneapolis, MN, USA). Hemoglobin A1c was measured with a Cobas Integra 800 automated biochemistry analyzer (Roche, Basel, Switzerland).

\section{Inflammatory marker measurement}

IL-6 and tumor necrosis factor receptor 1 (TNFR1) were measured using ELISA, following instructions provided by manufacturer (R\&D Systems, Minneapolis, MN, USA). Each sample was measured twice. The intra-assay coefficient of variation of each of these measurements was within acceptable ranges. An inflammation index score (IIS) was calculated according to a previous publication. ${ }^{7}$ The level of C-reactive protein (CRP) was measured by immunoturbidimetry (Beckman Coulter, Brea, CA, USA). 


\section{Adipokine and insulin pathway assay}

Circulating adiponectin, vaspin, leptin, and insulin-like growth factor 1 (IGF-1) were measured using ELISA, following instructions provided by manufacturer (R\&D Systems, Minneapolis, MN, USA).

\section{Statistical analysis}

Data were presented as mean \pm SD or mean rank or number and percentage. The difference in the characteristics between the two groups was evaluated by chi-squared test for categorical variable, independent $t$-test for continuous variables, and Kruskal-Wallis comparisons for the abnormally distributed continuous variables. Forward stepwise logistic regression was analyzed for the serum markers with frailty adjusted for age and chronic diseases. Pearson's or Spearman's correlation coefficients were calculated for the relationship of physical function with circulating cytokines and adipokines. Partial correlation analysis adjusted by age and sex was calculated. Statistical analyses were performed with SPSS (Chicago, IL, USA, version 11.0) or GraphPad Prism 7.0 software (GraphPad Software Inc., La Jolla, CA, USA). $P$-value was significant at $<0.05$.

\section{Results}

The characteristics of the 130 participants in this study are provided in Table 1. Compared with nonfrail adults, frail

Table I Characteristics of the nonfrail and frail older adults

\begin{tabular}{|c|c|c|c|c|}
\hline & Nonfrail $(n=90)$ & Frail $(n=40)$ & $\chi^{2 / t}$ & $P$-value \\
\hline Age (years) & $70.88 \pm 7.76$ & $77.13 \pm 8.95$ & 4.038 & $<0.001$ \\
\hline \multicolumn{5}{|l|}{ Gender (n, \%) } \\
\hline Male & $54(67.5)$ & $26(32.5)$ & & \\
\hline Female & $36(72.0)$ & $14(28.0)$ & 0.293 & 0.589 \\
\hline \multicolumn{5}{|l|}{ Education (years) } \\
\hline$\leq 8$ & $40(78.4)$ & II (2I.6) & & \\
\hline$>8$ & $48(63.2)$ & $28(36.8)$ & 3.346 & 0.067 \\
\hline \multicolumn{5}{|l|}{ Marriage (n, \%) } \\
\hline Live with spouse & $81(72.3)$ & $31(27.7)$ & & \\
\hline Live alone & $9(50.0)$ & $9(50.0)$ & 3.627 & 0.057 \\
\hline \multicolumn{5}{|l|}{ Hypertension (n, \%) } \\
\hline Yes & $58(63.0)$ & $34(37.0)$ & & \\
\hline No & $32(84.2)$ & $6(15.8)$ & 5.656 & 0.017 \\
\hline \multicolumn{5}{|l|}{ IHD (n, \%) } \\
\hline Yes & $36(60.0)$ & $24(40.0)$ & & \\
\hline No & $54(77.1)$ & $16(22.9)$ & 4.457 & 0.035 \\
\hline \multicolumn{5}{|l|}{ Diabetes (n, \%) } \\
\hline Yes & $25(61.0)$ & $16(39.0)$ & & \\
\hline No & $65(73.0)$ & $24(27.0)$ & 1.916 & 0.166 \\
\hline \multicolumn{5}{|l|}{ Stroke (n, \%) } \\
\hline Yes & $10(55.6)$ & $8(44.4)$ & & \\
\hline No & $80(71.4)$ & $32(28.6)$ & 1.834 & 0.176 \\
\hline $\mathrm{SBP}(\mathrm{mmHg})$ & $134.78 \pm 16.37$ & $139.78 \pm 21.84$ & 1.444 & 0.151 \\
\hline $\mathrm{DBP}(\mathrm{mmHg})$ & $75.16 \pm 11.59$ & $73.45 \pm 10.86$ & 0.789 & 0.431 \\
\hline $\mathrm{EF}(\%)$ & $64.48 \pm 7.21$ & $63.73 \pm 9.90$ & 0.535 & 0.594 \\
\hline BMI $\left(\mathrm{kg} / \mathrm{m}^{2}\right)$ & $25.8 \mathrm{I} \pm 3.68$ & $26.48 \pm 5.14$ & 0.846 & 0.399 \\
\hline WHR & $0.96 \pm 0.06$ & $0.96 \pm 0.05$ & 0.396 & 0.693 \\
\hline Grip strength (kg) & $27.19 \pm 10.07$ & $19.73 \pm 6.56$ & 4.195 & $<0.001$ \\
\hline Gait speed $(\mathrm{m} / \mathrm{s})$ & $0.99 \pm 0.50$ & $0.59 \pm 0.36$ & 4.342 & $<0.001$ \\
\hline Energy level & $6.78 \pm 1.92$ & $6.60 \pm 2.19$ & 0.158 & 0.877 \\
\hline
\end{tabular}

Note: Data are expressed as mean (SD) or $\mathrm{n}(\%)$

Abbreviations: $\mathrm{BMI}$, body mass index; $\mathrm{EF}$, ejection fraction; IHD, ischemic heart disease; WHR, waist-to-hip ratio. 
adults were more likely to be older. Chronic diseases such as hypertension and IHD were more common in frail older adults (all $P<0.05$ ), but no significantly differences were found in diabetes and stroke. No significant differences in sex, educational level, marital status, SBP, DBP, EF, BMI, waist-to-hip ratio, and energy level were found between the two groups. The percentage of slowness, fatigue, weight loss, weakness, and low activity was $41.1 \%, 58.3 \%, 5.4 \%, 55.9 \%$, and $30.8 \%$, respectively (data not shown).

As shown in Table 2, frail older adults had a higher BUN levels and lower HDL and HGB levels (all $P<0.05$ ) compared with nonfrail participants. No significant differences in TG, TC, LDL, Cr, ALT, AST, Hcy, PA, RBC, PLT, D dimer, BDNF, and HGA1b were observed between the two groups.

Frail participants displayed higher levels of inflammatory marker IL- 6 than nonfrail did $(0.71 \pm 0.28$ vs $0.58 \pm 0.22 \mathrm{pg} / \mathrm{mL}$, $P<0.01)$. The IIS was also higher in frail than those in nonfrail older adults $(4.47 \pm 0.34$ vs $4.33 \pm 0.26 \mathrm{pg} / \mathrm{mL}, P=0.01)$ (Figure 1). Although trends in TNFR1, CRP, fibrinogen, WBC, leptin, and insulin levels were increasing, the difference was not significant. Frail participants displayed higher levels of circulating adipokines such as adiponectin ( $\log , 3.91 \pm 0.13$ vs $3.83 \pm 0.20, P=0.01)$, vaspin $(0.89 \pm 0.27$ vs $0.79 \pm 0.25 \mathrm{ng} / \mathrm{mL}, P=0.04)$, and glucose (5.83 [5.20-7.40] vs $5.35[4.83 \pm 6.13] \mathrm{mmol} / \mathrm{L}, P=0.01)$ and lower levels of IGF-1
$(14.04 \pm 4.76$ vs $16.95 \pm 6.01 \mathrm{ng} / \mathrm{mL}, P<0.01)$ than nonfrail older adults (Figure 2).

Forward stepwise logistic regression of the serum markers with frailty showed that all of the blood markers were changed in frail participants: circulating IL-6, adiponectin, and glucose were elevated and IGF-1 and HDL were decreased after adjustment for age, hypertension, and IHD (Table 3).

We further explored the correlation between physical function with circulating cytokines and adipokines. Serum IL-6 levels were negatively correlated with both grip strength $(r=-0.19, P=0.03)$ and gait speed $(r=-0.18, P=0.04)$. Circulating adiponectin and leptin levels were adversely correlated with grip strength $(r=-0.23, P=0.01)$ and gait speed $(r=-0.19$, $P=0.03$ ), respectively (Figure 3 ). No significant differences were observed in the correlation between physical function and circulating inflammatory mediators and adipokines. The partial correlation coefficients adjusted by age and sex between these markers and physical function are presented in Table 4 . The only markers significantly related to physical function were IL-6 ( $r=-0.180, P=0.044)$ and adiponectin $(r=-0.195, P=0.029)$.

\section{Discussion}

Our study shows that higher levels of IL-6, adiponectin, vaspin, and glucose and lower levels of IGF-1 were associated

Table 2 Comparison of biochemical markers between frail and nonfrail old adults

\begin{tabular}{|c|c|c|c|c|}
\hline Total & Nonfrail group $(n=90)$ & Frail group $(n=40)$ & $t$-Value & $P$-value \\
\hline $\mathrm{TG}(\mathrm{mmol} / \mathrm{L})$ & $1.38(0.73)$ & $1.31(0.60)$ & 0.476 & 0.635 \\
\hline $\mathrm{TC}(\mathrm{mmol} / \mathrm{L})$ & $4.03(0.92)$ & $3.87(0.82)$ & 0.885 & 0.378 \\
\hline $\mathrm{HDL}-\mathrm{C}(\mathrm{mmol} / \mathrm{L})$ & $1.34(0.32)$ & $1.20(0.30)$ & 2.427 & 0.017 \\
\hline LDL-C (mmol/L) & $2.54(0.86)$ & $2.53(1.14)$ & 0.018 & 0.985 \\
\hline UA (mmol/L) & 335.27 (101.62) & $335.18(102.78)$ & 0.005 & 0.996 \\
\hline $\mathrm{Cr}(\mathrm{mmol} / \mathrm{L})$ & $71.10(23.15)$ & $71.79(25.54)$ & -0.152 & 0.880 \\
\hline $\mathrm{BUN}(\mathrm{mmol} / \mathrm{L})$ & $5.71(1.86)$ & $7.23(3.81)$ & -2.125 & 0.040 \\
\hline ALT (IU/L) & $19.88(15.90)$ & $20.95(17.42)$ & -0.341 & 0.733 \\
\hline AST (IU/L) & $23.16(13.93)$ & $23.16(13.93)$ & -0.213 & 0.832 \\
\hline Hcy (log) & $1.09(0.24)$ & $1.18(0.16)$ & -1.584 & 0.117 \\
\hline $\mathrm{PA}(\mathrm{mmol} / \mathrm{L})$ & $223.28(51.69)$ & $210.36(52.72)$ & 1.296 & 0.197 \\
\hline $\mathrm{RBC}\left(\times 10^{9} / \mathrm{L}\right)$ & $4.33(0.43)$ & $4.16(0.50)$ & 1.972 & 0.051 \\
\hline $\operatorname{PLT}\left(\times 10^{9} / \mathrm{L}\right)$ & $209.24(65.74)$ & $191.42(71.25)$ & 1.390 & 0.167 \\
\hline HGB $(g / L)$ & $133.49(15.62)$ & $125.38(18.42)$ & 2.584 & 0.011 \\
\hline$D$ dimer $(\mu g / m L)$ & $0.38(0.25-0.53)$ & $0.46(0.31-0.90)$ & 1.322 & 0.250 \\
\hline BDNF (ng/mL) & $2.19(0.73)$ & $2.29(0.70)$ & -0.715 & 0.476 \\
\hline $\mathrm{HbAlc}(\%)$ & $6.78(1.80)$ & 7.07 (I.37) & -0.672 & 0.504 \\
\hline
\end{tabular}

Note: Data are expressed as mean (SD) or median (IQR).

Abbreviations: ALT, alanine aminotransferase; AST, aspartate aminotransferase; BDNF, brain-derived neurotrophic factor; BUN, blood urea nitrogen; Cr, creatinine; HbAIc, hemoglobin A lc; Hcy, homocysteine; HDL, high-density lipoprotein; HGB, hemoglobin; LDL, low-density lipoprotein; PA, prealbumin; PLT, platelet; RBC, red blood cell count; TC, total cholesterol; TG, triglyceride; UA, uric acid. 

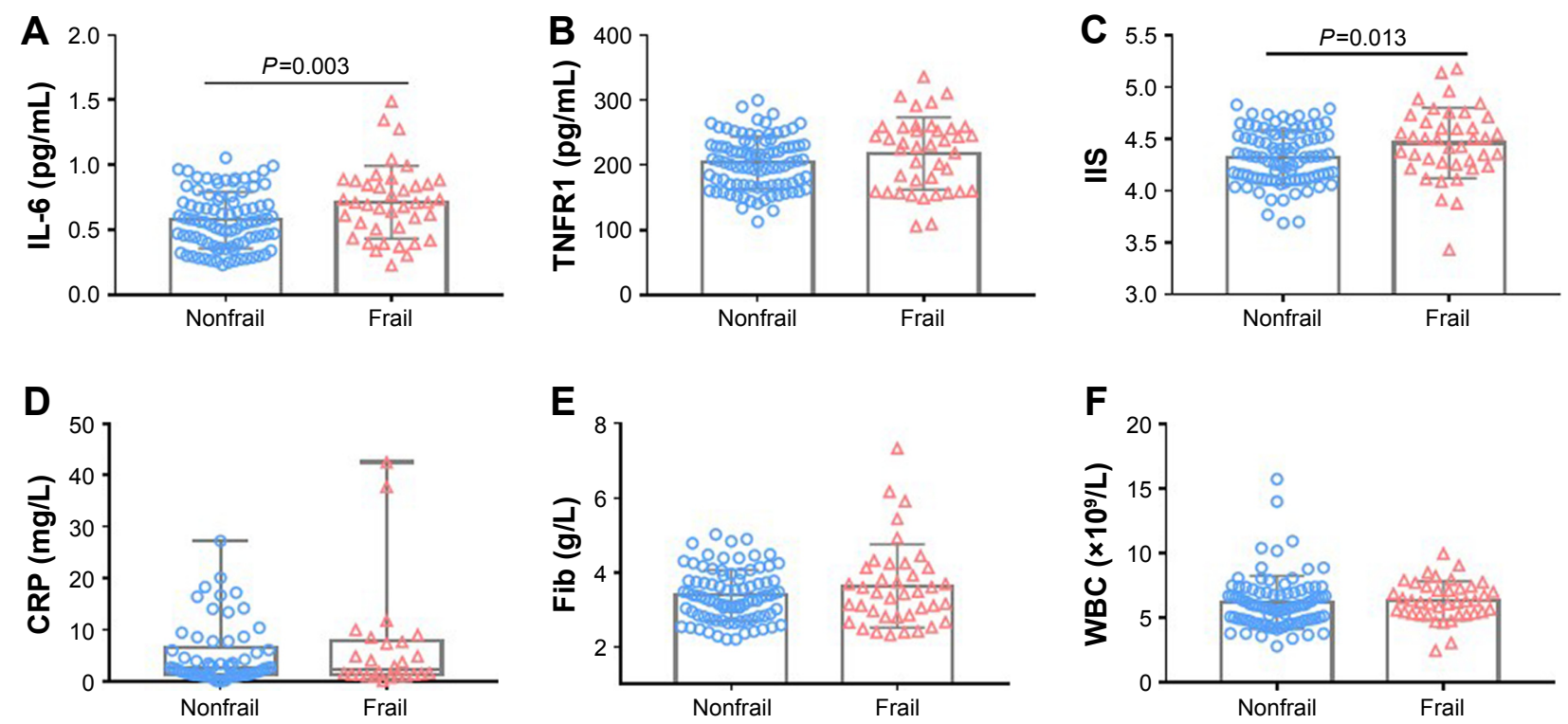

Figure I Comparison of inflammatory markers between frail and nonfrail old adults.

Notes: One hundred thirty older adults were divided into two groups: nonfrail group $(n=90)$ and frail group ( $n=40)$. Values in the figure were expressed as mean (SD) or median (IQR). Levels of IL-6 (A), TNFRI (B), IIS (C), CRP (D), Fib (E) and WBC (F) were compared between the two groups.

Abbreviations: CRP, C-reactive protein; Fib, fibrinogen; IIS, inflammation index score; TNFRI, tumor necrosis factor receptor I; WBC, white blood cell count.

with frailty, whereas serum IL-6 and adiponectin levels were negatively correlated with physical function. Our results suggest that inflammatory mediators IL-6 might be biomarkers for frailty and decreased physical function in older adults, which was consistent with other studies reporting the association between elevated IL-6 and frailty. ${ }^{8}$ A pro-inflammatory state is supposed to be at the center of a dynamic model of decreased physical function and physiological processes leading to frailty. ${ }^{2}$ IL-6 levels were independently associated with worse functional status, progression of age-related diseases, disability, morbidity, and increased mortality rates in older adults. Particularly, low-grade, chronic, and systemic inflammation is two- to threefold higher in older individuals. ${ }^{9}$ Serum IL-6 levels were negatively correlated with both grip
A
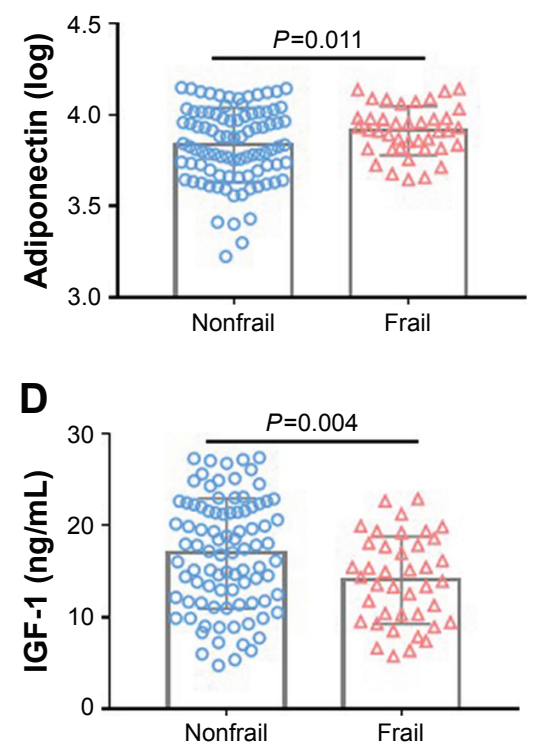

B

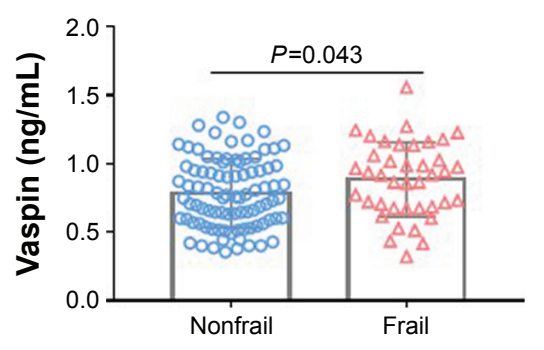

E

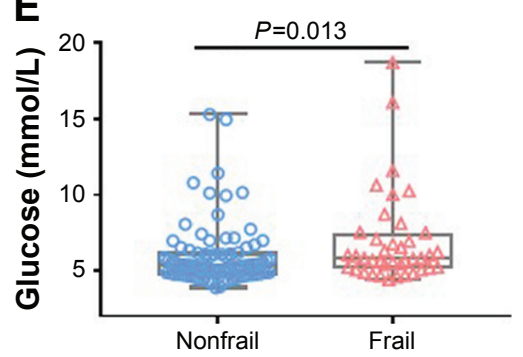

C

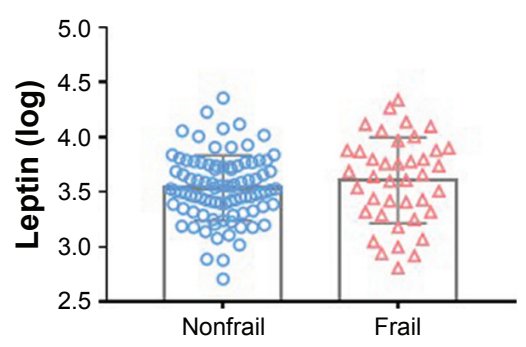

$\mathbf{F}$

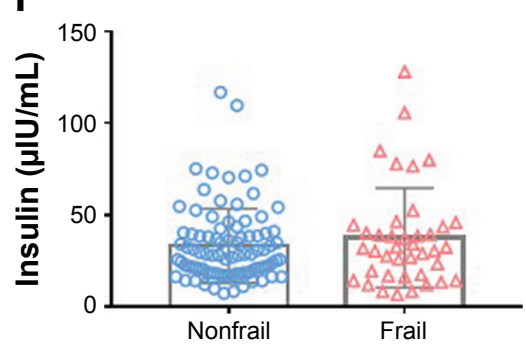

Figure 2 Comparison of circulating adipokines and insulin pathway between frail and nonfrail old adults.

Notes: One hundred thirty older adults were divided into two groups: nonfrail group $(n=90)$ and frail group ( $=40)$. Values in the figure were expressed as mean (SD) or median (IQR). Levels of adiponectin (A), vaspin (B), leptin (C), IGF-I (D), glucose (E) and insulin (F) were compared between the two groups.

Abbreviation: IGF-I, insulin-like growth factor I. 
Table 3 Forward stepwise logistic regression for the serum markers with frailty

\begin{tabular}{|c|c|c|c|c|c|c|}
\hline & \multicolumn{3}{|l|}{ Model I } & \multicolumn{3}{|l|}{ Model 2} \\
\hline & $\operatorname{Exp}(B)$ & $95 \% \mathrm{Cl}$ & $P$-value & $\operatorname{Exp}(B)$ & $95 \% \mathrm{Cl}$ & $P$-value \\
\hline IL-6 & 10.438 & $2.057-52.970$ & 0.005 & 6.493 & $1.167-36.109$ & 0.033 \\
\hline IIS & 5.523 & I.383-22.054 & 0.016 & - & - & - \\
\hline Adiponectin (log) & 11.225 & $1.220-103.290$ & 0.033 & 29.730 & $2.238-394.887$ & 0.010 \\
\hline Vaspin & 4.517 & $1.031-19.792$ & 0.045 & - & - & - \\
\hline IGF-I & 0.912 & $0.85 I-0.978$ & 0.009 & 0.901 & $0.835-0.972$ & 0.007 \\
\hline Glucose & 1.168 & $1.001-1.364$ & 0.048 & 1.185 & $1.003-1.401$ & 0.046 \\
\hline $\mathrm{HDL}$ & 0.203 & $0.053-0.774$ & 0.020 & 0.210 & $0.052-0.842$ & 0.028 \\
\hline BUN & 1.233 & I.040-I.462 & 0.016 & - & - & - \\
\hline HGB & 0.970 & $0.947-0.994$ & 0.014 & - & - & - \\
\hline
\end{tabular}

Notes: Model I: no adjust. Model 2: adjusted for age, hypertension, and chronic heart disease.

Abbreviations: BUN, blood urea nitrogen; HDL, high-density lipoprotein; HGB, hemoglobin; IGF-I, insulin-like growth factor I; IIS, inflammation index score.

strength and gait speed in this study, which indicates that inflammation may be the molecular mechanism underlying physical frailty and inflammation. We did not find the difference in CRP between the two groups or its correlation with physical function; however, a recent study found that CRP was predictive of worse grip strength. ${ }^{10}$

Circulating adiponectin was higher in frail older adults and adversely correlated with grip strength in this study.
Adiponectin is a multifunctional adipokine with insulinsensitizing, anti-inflammatory, and anti-atherosclerotic functions. An "adiponectin paradox" phenomenon was found as an evidence in the association between circulating adiponectin and metabolic dysfunction; on one hand, decreased adiponectin levels were associated with a higher frequency of type 2 diabetes and metabolic syndrome. ${ }^{11}$ Moreover, centenarians have higher adiponectin levels associated with
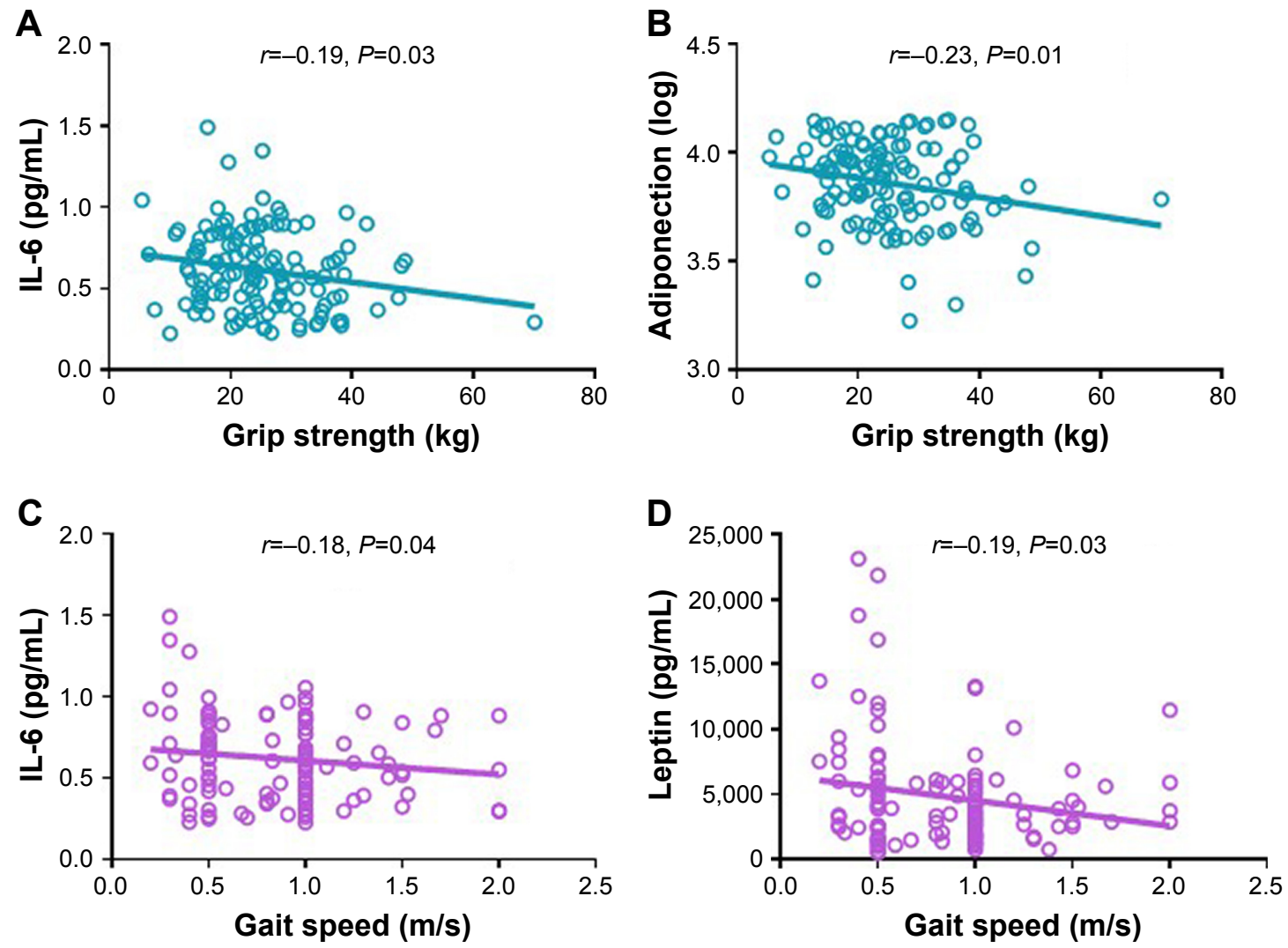

Figure 3 Correlation between physical function and circulating inflammatory markers and adipokines.

Notes: Pearson's correlation coefficients were calculated between grip strength and IL-6 (A) and adiponectin (B). Spearman's correlation coefficients were calculated between gait speed and IL-6 (C) and leptin (D) in I30 older adults. 
Table 4 Age- and sex-adjusted partial correlation coefficients between serum markers and physical function

\begin{tabular}{l|l|l|l|l}
\hline \multirow{2}{*}{} & \multicolumn{2}{|l|}{ Grip strength (kg) } & \multicolumn{2}{l}{ Gait speed (m/s) } \\
\cline { 2 - 5 } & $\boldsymbol{r}$ & $\boldsymbol{P}$-value & $\boldsymbol{r}$ & $\boldsymbol{P}$-value \\
\hline IL-6 & -0.180 & 0.044 & $-0.13 \mathrm{I}$ & $0.15 \mathrm{I}$ \\
Adiponectin (log) & -0.195 & 0.029 & -0.060 & 0.510 \\
Vaspin & -0.084 & 0.350 & -0.050 & 0.586 \\
IGF-I & 0.100 & 0.267 & 0.171 & 0.061 \\
Leptin & -0.009 & 0.921 & -0.112 & 0.221 \\
\hline
\end{tabular}

Abbreviation: IGF-I, insulin-like growth factor I.

longevity. Further studies showed that adiponectin levels were negatively correlated with visceral fat accumulation; ${ }^{12}$ one the other hand, high adiponectin levels are associated with a low risk of type 2 diabetes ${ }^{13}$ and are an indicator of the incidence of cardiovascular diseases, physical disability, and higher mortality rate. ${ }^{14}$ Studies focusing on frailty found that high adiponectin level was associated with frailty status and correlated with an increasing number of frailty components in older adults; ${ }^{15}$ however, in a frail mouse model plasma, levels of adiponectin and leptin were decreased. ${ }^{16}$ Adiponectin resistance involving insulin resistance, inflammation, and metabolic dysregulation might be a key mechanism underlying this phenomenon.

Although we did not find a significant correlation between vaspin and physical function, its levels were high in frail participants. No studies have explored the vaspin levels in frail participants yet. Vaspin is a visceral adipose tissuederived serine protease inhibitor with insulin-sensitizing effects, which is associated with obesity and insulin resistance. Patients with type 2 diabetes mellitus and low serum vaspin levels were at risk of acute cardiac syndrome. ${ }^{17}$ Other studies found that vaspin level increased with aging and was associated with parasympathetic activity even in the absence of metabolic syndrome. ${ }^{18}$ In addition, a study found that vaspin cannot be used as an independent marker for the presence of coronary artery disease in the general population. ${ }^{19}$

Frail older adults displayed higher glucose and BUN levels and lower IGF-1, HDL, and HGB levels compared with nonfrail participants, which indicates that the former had more insulin resistance and probably worse nutritional status. IGF-1 is an anabolic hormone that plays an active role in the maintenance of muscle mass and strength, preventing apoptosis and protection from oxidative stress. It is a possible indicator for hormonal, inflammatory, or nutritional pathway to frailty. Serum IGF-1 is positively correlated with physical performance, ${ }^{20}$ thigh muscle area and density, ${ }^{21}$ knee extensor strength, and self-reported difficulty in several mobility-related tasks ${ }^{22}$ and negatively associated with health outcomes. ${ }^{23}$ Decreased serum IGF-1 levels are independently related to frailty in older adults, ${ }^{24}$ consequently associated with reduced muscle size, strength, diminished protein synthesis, and increased muscle cell apoptosis. ${ }^{25}$ IGF-1 may be assumed as an ideal cross-road of nutritional, hormonal, and inflammatory pathways to frailty. ${ }^{26}$ Both the secretion and biological actions of IGF-1 are modulated by the main pro-inflammatory cytokines. The negative effect of IL- 6 on muscle function is through IGF-1, ${ }^{27}$ while the effect of IGF-1 on muscle function depends on IL-6 levels. ${ }^{28}$

There are several limitations in this study. First, the cross-sectional study design could not explain the causal relationship between circulating inflammatory mediators or adipokines and frailty; thus, further follow-up on changes in physical function is needed. Second, the observed association might be influenced by confounders, due to the small sample size. We were unable to use multilevel logistic regression analysis to adjust several variables and biases related with sample that should be considered. The $r$ value of the correlation was small, partly due to the small sample size. Lastly, inflammatory cytokines and adipokines are dynamic, which may change over time; thus, multiple time measurement should be conducted in further studies.

\section{Discussion}

To the best of our knowledge, this study is the first to show the association of circulating IL-6 and adiponectin with frailty and physical function in a Chinese older population. These preliminary findings suggest that adipokines could be considered as potential biomarkers for decreased physical function in older adults. Longitudinal studies are needed to evaluate the causal relationship between inflammation and adipokines and prefrailty and frailty, as well as their predictive role in the onset of frailty in older individuals. Our findings provide a rationale of using biological markers in the identification, prevention, and treatment of frailty.

\section{Acknowledgments}

This work was supported by Milstein Medical Asian American Partnership Foundation Project Award in Geriatrics (2018) and Beijing Municipal Health Bureau Research Fund (Jing 17-12).

\section{Disclosure}

The authors report no conflicts of interest in this work. 


\section{References}

1. Kirkland JL. Translating advances from the basic biology of aging into clinical application. Exp Gerontol. 2013;48(1):1-5.

2. Fried LP, Tangen CM, Walston J, et al. Frailty in older adults: evidence for a phenotype. J Gerontol A Biol Sci Med Sci. 2001;56(3):M146-M157.

3. López-Otín C, Blasco MA, Partridge L, Serrano M, Kroemer G. The hallmarks of aging. Cell. 2013;153(6):1194-1217.

4. Lio D, Scola L, Crivello A, et al. Inflammation, genetics, and longevity: further studies on the protective effects in men of IL-10-1082 promoter SNP and its interaction with TNF-alpha-308 promoter SNP. J Med Genet. 2003;40(4):296-299.

5. Nagasawa M, Takami Y, Akasaka H, et al. High plasma adiponectin levels are associated with frailty in a general old-old population: The Septuagenarians, Octogenarians, Nonagenarians Investigation with Centenarians study. Geriatr Gerontol Int. 2018;18(6):839-846.

6. Wu C, Smit E, Xue QL, Odden MC. Prevalence and correlates of frailty among community-dwelling Chinese older adults: The China Health and Retirement Longitudinal Study. J Gerontol A Biol Sci Med Sci. 2017;73(1):102-108.

7. Varadhan R, Yao W, Matteini A, et al. Simple biologically informed inflammatory index of two serum cytokines predicts 10 year all-cause mortality in older adults. J Gerontol A Biol Sci Med Sci. 2014;69(2): 165-173.

8. de Martinis M, Franceschi C, Monti D, Ginaldi L. Inflammation markers predicting frailty and mortality in the elderly. Exp Mol Pathol. 2006; 80(3):219-227.

9. Calçada D, Vianello D, Giampieri E, et al. The role of low-grade inflammation and metabolic flexibility in aging and nutritional modulation thereof: a systems biology approach. Mech Ageing Dev. 2014;136-137: 138-147.

10. Schaap LA, Pluijm SM, Deeg DJ, Visser M. Inflammatory markers and loss of muscle mass (sarcopenia) and strength. Am J Med. 2006;119(6):526.e9-526.e17.

11. Liu C, Feng X, Li Q, Wang Y, Li Q, Hua M. Adiponectin, TNF- $\alpha$ and inflammatory cytokines and risk of type 2 diabetes: a systematic review and meta-analysis. Cytokine. 2016;86:100-109.

12. Ryo M, Nakamura T, Kihara S, et al. Adiponectin as a biomarker of the metabolic syndrome. Circ J. 2004;68(11):975-981.

13. Li S, Shin HJ, Ding EL, van Dam RM. Adiponectin levels and risk of type 2 diabetes: a systematic review and meta-analysis. JAMA. 2009; 302(2):179-188

14. Persson J, Folkersen L, Ekstrand J, et al. High plasma adiponectin concentration is associated with all-cause mortality in patients with carotid atherosclerosis. Atherosclerosis. 2012;225(2):491-496.
15. Tsai JS, Wu CH, Chen SC, et al. Plasma adiponectin levels correlate positively with an increasing number of components of frailty in male elders. PLoS One. 2013;8(2):e56250.

16. Westbrook RM, Yang HL, Langdon JM, et al. Aged interleukin-10tm1Cgn chronically inflamed mice have substantially reduced fat mass, metabolic rate, and adipokines. PLoS One. 2017;12(12):e0186811.

17. Sathyaseelan AJ, Adole PS, Wyawahare M, Saya RP. Assessment of serum VASPIN levels among type 2 diabetes mellitus patients with or without acute coronary syndrome. J Clin Diagn Res. 2016;10(12): BC07-BC10.

18. Dimova R, Tankova T, Kirilov G, Chakarova N, Dakovska L, Grozeva G. Is vaspin related to cardio-metabolic status and autonomic function in early stages of glucose intolerance and in metabolic syndrome? Diabetol Metab Syndr. 2016;8(1):46.

19. Stančík M, Ságová I, Kantorová E, Mokáň M. The role of vaspin as a predictor of coronary angiography result in SCAD (stable coronary artery disease) patients. BMC Cardiovasc Disord. 2017;17(1):117.

20. van Nieuwpoort IC, Vlot MC, Schaap LA, Lips P, Drent ML. The relationship between serum IGF-1, handgrip strength, physical performance and falls in elderly men and women. Eur J Endocrinol. 2018;179(2): 73-84.

21. Colbert LH, Rosen $\mathrm{CJ}$, Goodpaster $\mathrm{BH}$, et al. Insulin-like growth factor-1. J Am Geriatr Soc. 2004;52(11):1962-1963.

22. Cappola AR, Bandeen-Roche K, Wand GS, Volpato S, Fried LP. Association of IGF-I levels with muscle strength and mobility in older women. J Clin Endocrinol Metab. 2001;86(9):4139-4146.

23. Kaplan RC, Mcginn AP, Pollak MN, et al. Total insulinlike growth factor 1 and insulinlike growth factor binding protein levels, functional status, and mortality in older adults. J Am Geriatr Soc. 2008;56(4):652-660.

24. Doi T, Makizako H, Tsutsumimoto K, et al. Association between insulin-like growth factor-1 and frailty among older adults. $J$ Nutr Health Aging. 2018;22(1):68-72.

25. Perrini S, Laviola L, Carreira MC, Cignarelli A, Natalicchio A, Giorgino F. The GH/IGF1 axis and signaling pathways in the muscle and bone: mechanisms underlying age-related skeletal muscle wasting and osteoporosis. J Endocrinol. 2010;205(3):201-210.

26. Maggio M, de Vita F, Lauretani F, et al. IGF-1, the cross road of the nutritional, inflammatory and hormonal pathways to frailty. Nutrients. 2013; 5(10):4184-4205

27. Lazarus DD, Moldawer LL, Lowry SF. Insulin-like growth factor-1 activity is inhibited by interleukin-1 alpha, tumor necrosis factor-alpha, and interleukin-6. Lymphokine Cytokine Res. 1993;12(4):219-223.

28. Barbieri M, Ferrucci L, Ragno E, et al. Chronic inflammation and the effect of IGF-I on muscle strength and power in older persons. Am J Physiol Endocrinol Metab. 2003;284(3):E481-E487.
Clinical Interventions in Aging

\section{Publish your work in this journal}

Clinical Interventions in Aging is an international, peer-reviewed journal focusing on evidence-based reports on the value or lack thereof of treatments intended to prevent or delay the onset of maladaptive correlates of aging in human beings. This journal is indexed on PubMed Central, MedLine,
Dovepress

CAS, Scopus and the Elsevier Bibliographic databases. The manuscript management system is completely online and includes a very quick and fair peer-review system, which is all easy to use. Visit http://www.dovepress. com/testimonials.php to read real quotes from published authors. 